\title{
La lutte contre la corrosion du matériel des usines marémotrices
}

\section{Preventing corrosion on tidal plant equipment}

\author{
PAR S. LICHERON
}

SERVICE D'ÉTUDES SUR L'UTILISATION DES MARËES

\begin{abstract}
Recherches entreprises par Electricité de France depuis 1955 sur les lieux mêmes du futur barrage de la Rance, sur la protection du matériel des usines marémotrices contre la corrosion par l'eau de mer ou l'atmosphère saline :

- Etude des comportements des revêtements anticorrosifs exposés d̀ l'immersion prolongée, à l'immersion-émersion, à l'air marin;

- Comportement de dinerses nuances d'aciers inoxydables et de bronze d'aluminium $\dot{a}$ l'eau de mer.

Essais effectués, résultats obtenus et confirmés par le comportement d'un groupe industriel mis en service en octobre 1959.
\end{abstract}

\begin{abstract}
Research carried out by Electricité de France at the site of the future Rance barrage since 1955 has been concerned with the problem of protecting tidal plant equipment against the corrosive action of sea air and salt water. The following work has been carried out:

i) A study of the behaviour of various anticorrosive covering during prolonged immersion or immersion followed by emersion, and when exposed to the sea air;

ii) A study of the resistance of various grades of stainless steel and aluminium bronze to sea water corrosion.

The results of the tests were subsequently confirmed by experience with a prototype unit, which has now been operating since 1959.
\end{abstract}

La réalisation industrielle d'une usine marémotrice pose un problème particulierement important : celui du choix des matériaux et de leur protection contre la corrosion marine. Pour tenter de le résoudre dans le cas particulier de l'usine de la Rance, d'importantes séries d'essais ont été entreprises par Electricité de France dès 1955 au Service d'Etudes sur l'Utilisation des Marées (S.E.U.M.) à Saint-Servan. Coordonnés par un groupe de travail, en liaison étroite avec les laboratoires d'Electricité et du Gaz de France, des principaux métallurgistes français et de certains organismes spécialisés, les essais, effectués sur les lieux mêmes et dans des condi- tions aussi proches que possible des conditions du service réel, ont porté sur les points suivants:

- Etude des comportements des revêtements anticorrosifs des métaux à la corrosion marine;

- Comportement de diverses nuances d'aciers et d'alliages cuivreux à l'eau de mer.

Le présent rapport rend compte, pour chacun de ces problèmes;

- des essais effectués et des moyens mis en œuvre pour les conduire,

- des résultats obtenus. 


\section{A. - COMPORTEMENTS DES REVETEMENTS ANTICORROSIFS DES METAUX}

\section{I. - PROGRAMME ET MOYENS D'ESSAIS}

Le programme d'essai a porté sur l'étude des comportements des revêtements anticorrosion et antisalissures exposés :

- à l'immersion prolongée,

- à l'immersion-émersion,

- à l'atmosphère marine.

Ce programme de base se complète par des études de détails :

- Influence des formes du support;

- Influence de l'épaisseur du film de peinture;

- Essais sur circuit hydraulique dans des conditions d'utilisation;

- Tests de résistance sous protection cathodique effectués au Laboratoire G.D.F.

\section{a) Stations D'Essais.}

Poür l'immersion prolongée, nous disposions jusqu'en 1961 d'une sapine implantée à la cote-4 CM sur l'axe même du futur barrage. Cette sapine était étudiée pour recevoir des éprouvettes sous forme de plaques et de tubes. Les courants à cet endroit atteignaient 3 noeuds au maximum. Depuis le $1^{\text {er }}$ janvier 1961, elle a été remplacée par un radeau (fig. 1) mouillé en eau vive au sud de l'emplacement précédent.

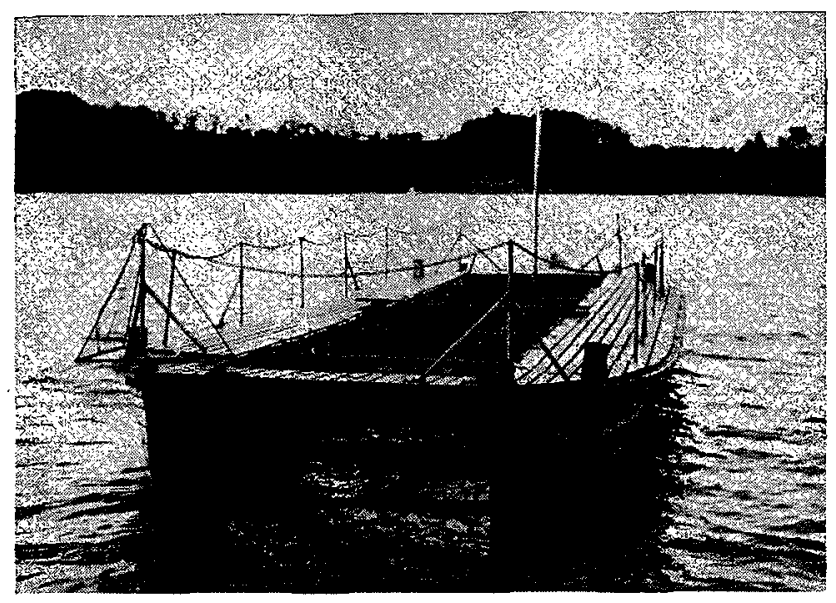

FIG. 1

Radeau mouillé en eau vive.

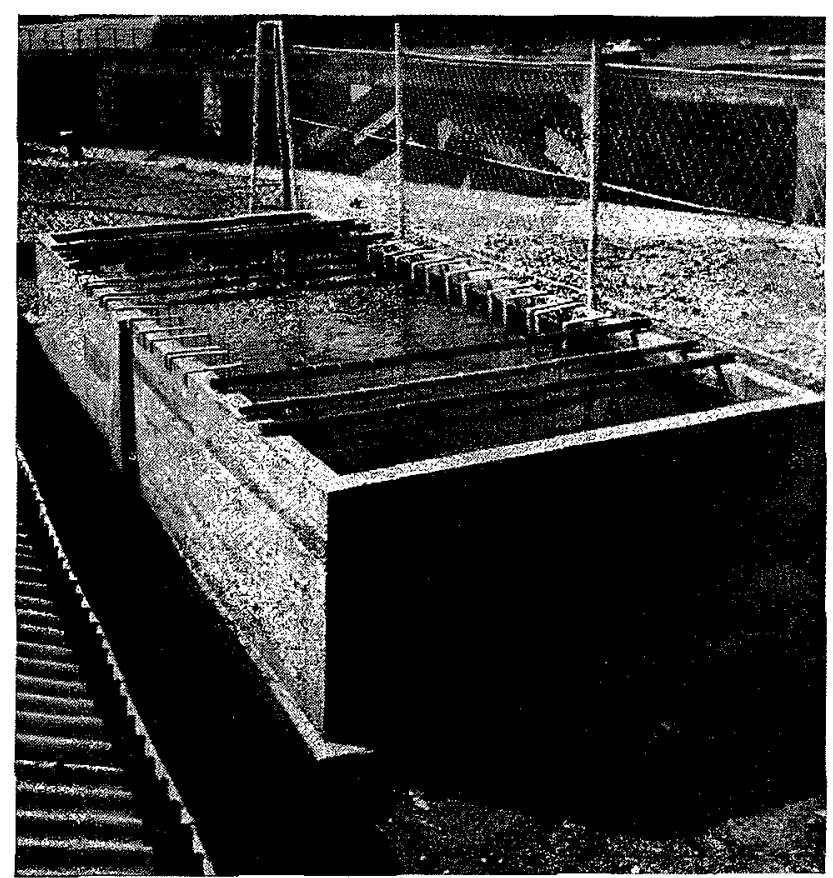

Fig. 2

Bac à circulation permanente d'eau de mer.

Une seconde station, dite de «pré-essai» (fig. 2), située près de nos bureaux, a été aménagée. Elle se compose d'un bassin en ciment de 5500 litres alimenté en permanence d'eau de mer naturelle à un débit de $30 \mathrm{~m}^{3}$ /heure.

Pour l'immersion-émersion, nous disposons d'une estacade (fig. 3) située à la cote de la mini-

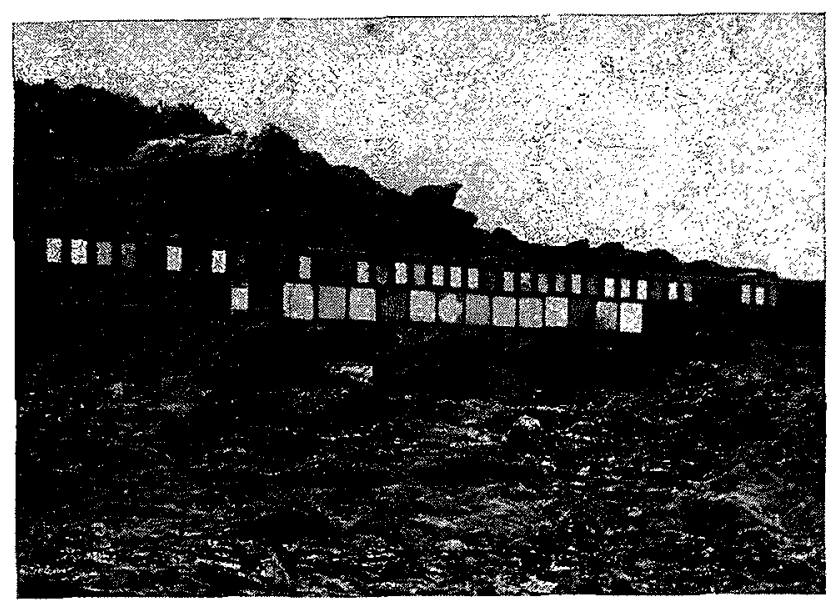

Fig. 3

Estacade située à la cote de la mi-marée en Ránce. 


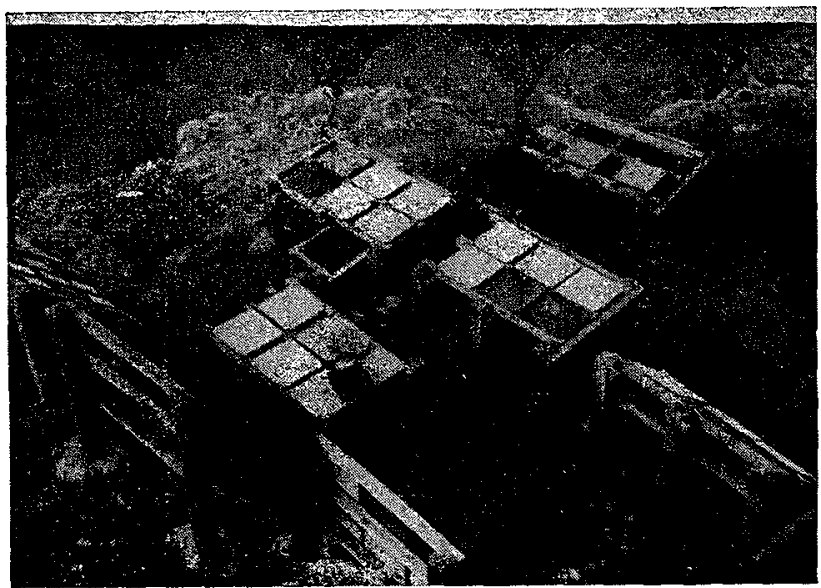

FIG. 4

Exposition à l'air marin.

marée, susceptible de recevoir des plaques éprouvettes. Cette installation, initialement implantée sur l'îlot Chalibert (point d'appui du barrage), a été transplantée en janvier 1961 sur l'île Chevret dans le sud.

Pour les études à l'atmosphère marine, nous utilisons le Centre de contrôle de corrosion de Cézembre (fig. 4). Cet emplacement, situé à la cote $+20 \mathrm{CM}$, fait face au noroît et se trouve particulièrement exposé aux embruns. Les éprouvettes sont placées sur des pupitres sous trois expositions : verticale, horizontale, $45^{\circ}$.

\section{b) Préparation des ÉProuvettes.}

La majeure partie des éprouvettes a étẻ revêtue par nos soins dans un atelier aménagé à cet effet. Les métallisations et quelques revêtements à chaud ou spéciaux ont été traités chez le fournisseur.

Les éprouvettes utilisées sont en acier ordinaire, leur dimension est de $300 \times 200 \times 3 \mathrm{~mm}$.

La préparation des éprouvettes comporte les opérations suivantes :

- Sablage à blanc au corindon calibre $\mathrm{C}$;

- Application de la première couche dans les trois heures qui suivent;

- Application des autres couches conformément aux indications du fournisseur;

- Contrôle de l'épaisseur du revêtement à l'aide d'un appareil magnétique.

\section{c) Observations et Relevés.}

Les éprouvettes sont examinées au minimum tous les deux mois. L'évolution des revêtements est notée sur fiches et suivie par dessins ou photos en noir et en couleur, à la lumière rasante.

\section{II. - RESULTATS DES ESSAIS}

Au total, nos stations en Rance ont permis de mener des essais sur :

- 110 revêtements en immersion totale, dont 41 à finition antifouling;

- 114 revêtements en immersion-émersion, dont 35 à finition antifouling;

- 102 revêtements à l'air salin et aux embruns.

La nature de ces divers revêtements apparaît sur le tableau $\mathrm{I}$.

Par ailleurs, étant donné le grand nombre de revêtements proposés par les fabricants de pelnture, notre station de «pré-essais》 installée depuis fin 1959 nous a permis d'éliminer 51 revêtements sur 72 essayés.

a) Comportement EN IMMERsion PRolongée.

Ce milieu est le plus agressif.

\section{TABlead I}

\begin{tabular}{|c|c|c|c|}
\hline \multirow{2}{*}{ SYSTÈMES } & \multicolumn{3}{|c|}{$\begin{array}{l}\text { Nombre dE REVÊtEMENTS } \\
\text { MIS EN ESSAI EN RANCE }\end{array}$} \\
\hline & $\begin{array}{l}\text { Immer- } \\
\text { sion }\end{array}$ & Marnage & Air salin \\
\hline Bitumineux, . . . . . & 9 & 9 & 7 \\
\hline Caoutchouc. . .... & 12 & 12 & 17 \\
\hline Epoxy. .......... & 9 & 9 & 9 \\
\hline Glycérophtalique. . . . & 2 & 2 & 7 \\
\hline Isocyanate. . . . . . . & 5 & 5 & 5 \\
\hline Oléosynthétique. . . . & 0 & 0 & 7 \\
\hline Métallisation. . . . . . & 13 & 11 & 9 \\
\hline Phénolique. . . . . . & $\mathbf{3}$ & 2 & 1 \\
\hline Stratifié. . . . . . . . & 2 & 2 & 0 \\
\hline Vinylique. . . . . . . & 46 & 54 & 32 \\
\hline Zinc. ......... & 9 & 8 & 8 \\
\hline
\end{tabular}




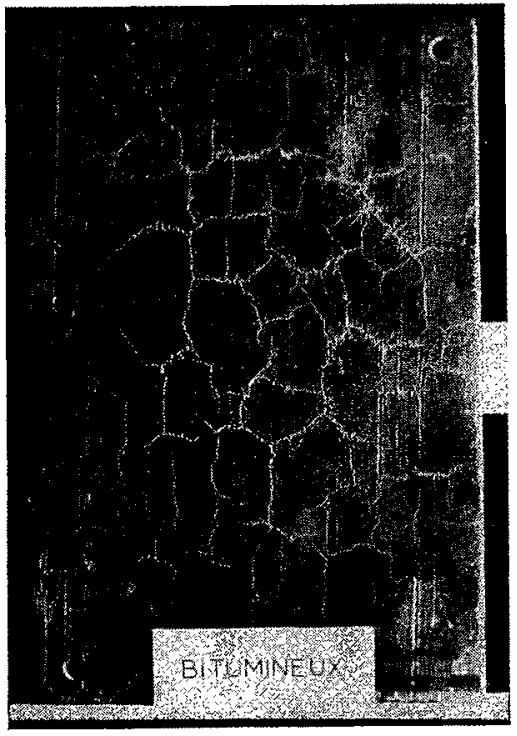

FiG. 5

Après 1 an d'immersion.

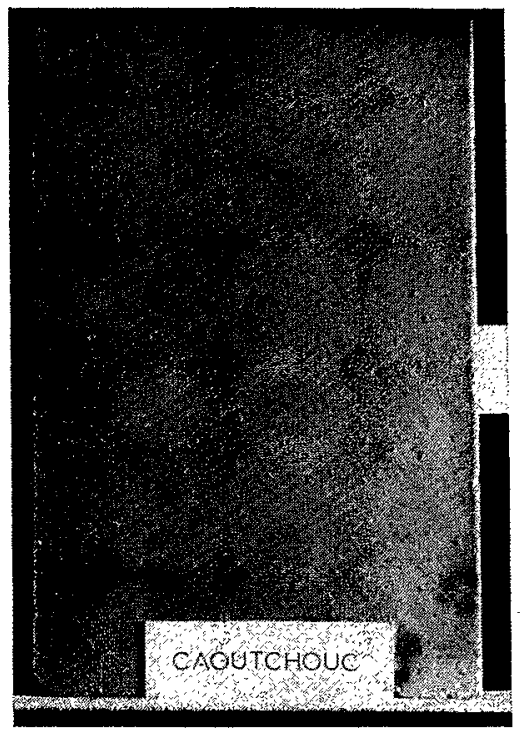

FIG, 6

Après 1 an d'immersion.

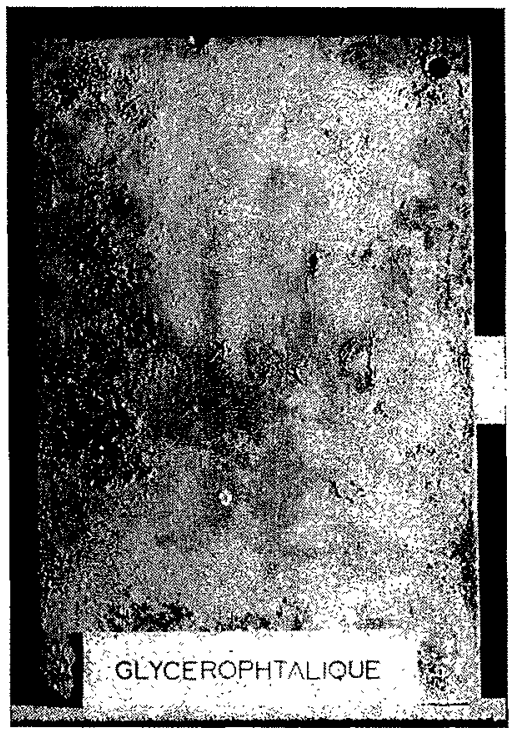

FrG. 7

Après 1 an d'immersion.
Les revêtements bitumineux (fig. 5) sont éliminés à 1 an pour craquelures et rouille; les premières attaques apparaissent entre 1 et 5 mois.

Les revêtements caoutchouc (fig. 6) disparaissent également à 1 an par cloquage et manque d'adhérence.

Les produits glycérophtaliques (fig. 7) ont aussi une tenue inférieure à 1 an.
Les peintures à poudre de zinc (fig. 8) ont une durée brève, moins d'un an si elles ne sont pas recouvertes d'une finition; mais malgré cette finition, le phénomène important est le cloquage qui apparaît avant 6 mois. Ce sont des cloques sèches qui font s'exfolier les différentes couches les unes par rapport aux autres, mais qui peuvent aller jusqu'au métal.

La tenue convenable des isocyanates (fig. 9) est de l'ordre de 3 ans; les premières attaques

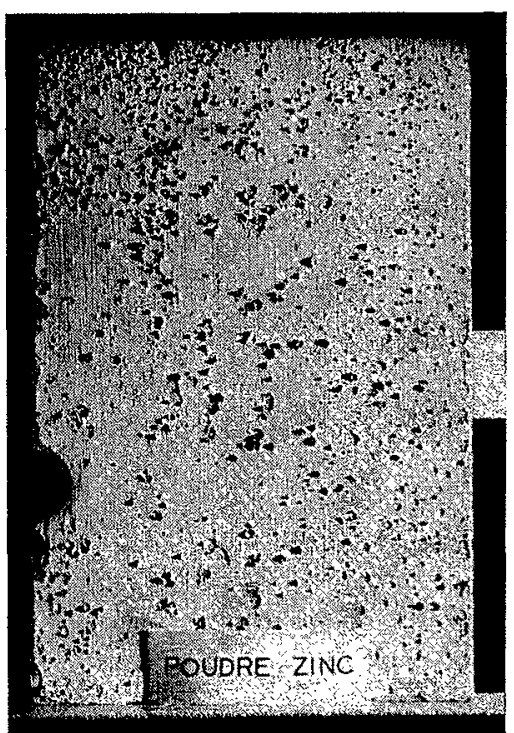

Fig. 8

Après 1 an d'immersion.

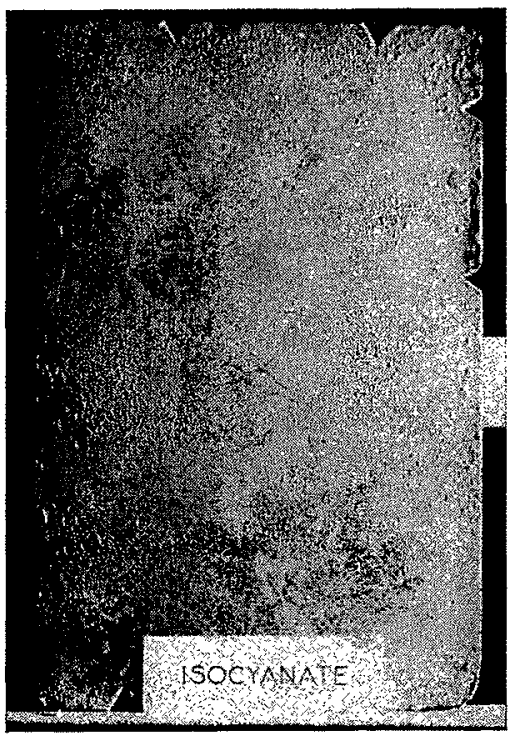

FiG. 9

Après 4 ans d'immersion.

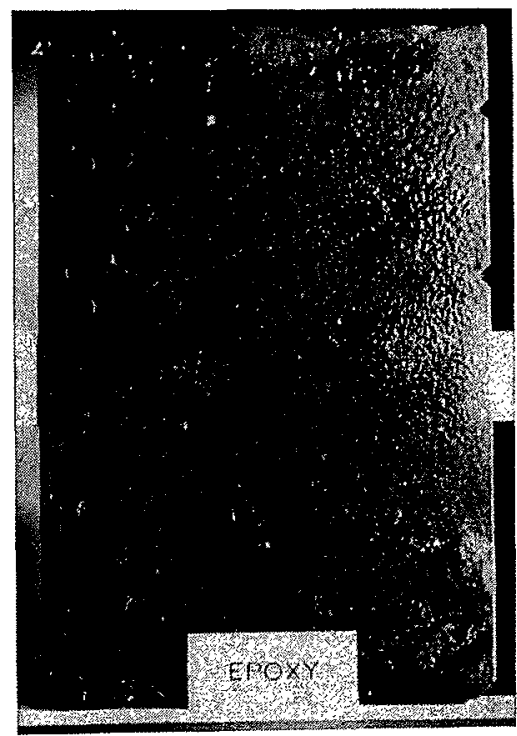

FIG. 10

Après 4 ans et demi d'immersion. 


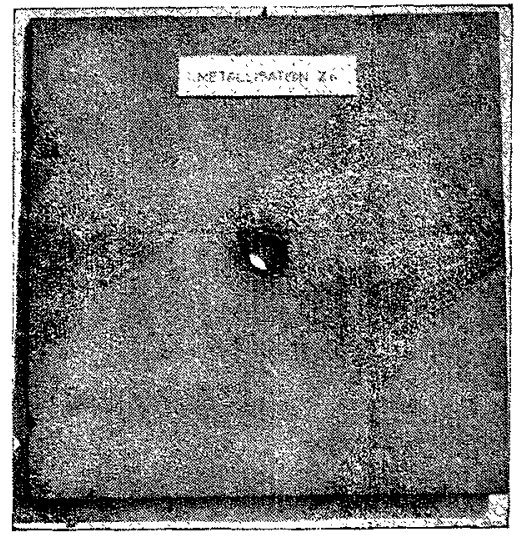

FIG. 11

Après 4 ans et demi d'immersion.

apparaissent entre le $5^{\circ}$ et $10^{\circ}$ mois sous forme de petites cloques qui évoluent lentement en décollements.

Parmi les produits epoxy (fig. 10) en essais, la plupart ont été exposés récemment (1 an); cependant deux produits mis en essais en 1957 ont résisté convenablement 3 ans.

Les métallisations au zinc (fig. 11), malgré qu'elles soient l'objet d'attaques localisées de la couche de zinc par pitting, puis de cavités remplies de poudre blanche (oxychlorure de zinc), se comportent bien. Certaines métallisations sont encore en essais depuis février 1956.
Ce système présente l'avantage de remédier aux détériorations éventuelles du revêtement par déplacement sous l'effet d'électrolyse du zinc sur la blessure. Et, par suite, l'utilisation de ce revêtement sur un ensemble comportant des métaux nobles est à proscrire, car le zinc se consommerait très rapidement

Les métallisations aluminium se comportent également bien, mais présentent une moins bonne adhérence sur le métal de base.

Les bonnes peintures vingliques résistent au moins 3 ans.

Le phénomène général le plus important est le cloquage; il apparaît, suivant les cas, de 3 à 6 mois après l'immersion et s'accompagne souvent d'une perte d'adhérence au voisinage des cloques. Ces cloques sont remplies de liquide dont le $\mathrm{pH}$ est de l'ordre de 13; si elles sont ouvertes par accident, elles donnent, par la suite, un nodule de rouille.

Une excellente peinture française est encore «belle» après 4 ans $1 / 2$. Tous les essais confirment cette supériorité (fig. 12).

Les métallisations + peintures vinyliques (fig. 13) bénéficient des avantages des deux systèmes, mais la proscription faite au sujet des métallisations zinc dans un ensemble comportant des métaux nobles est encore valable.

Les systèmes à finition antifouling présentent une meilleure tenue que ceux à finition inerte sur lesquels se déposent algues et balanes. Ce

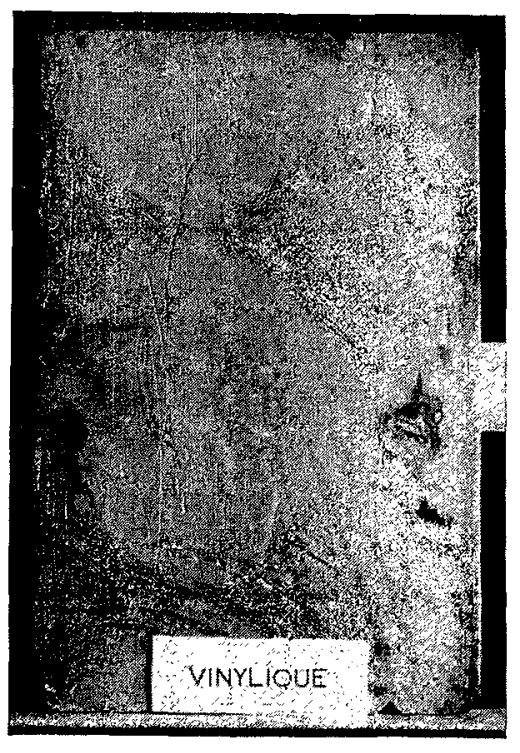

FIG. 12

Après 4 ans et demi d'immersion.

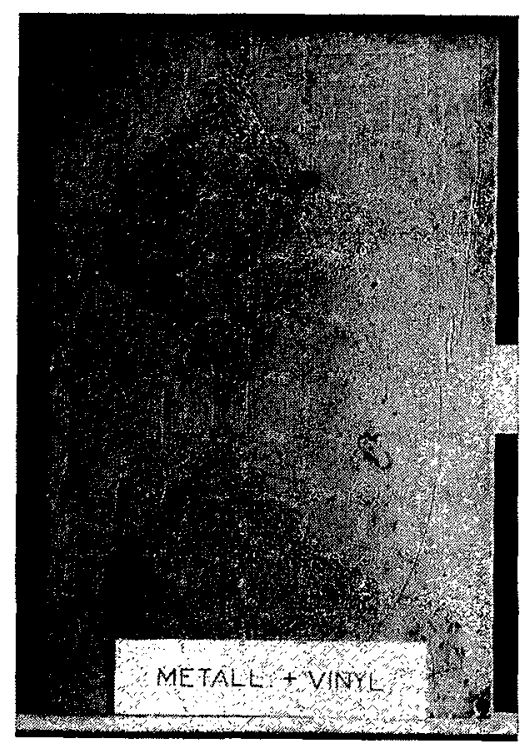

Fig. 13

Après 4 ans et demi d'immersion. 
fait peut s'expliquer par l'action de la corrosion biologique et par l'aération différentielle que peuvent occasionner les organismes se fixant sur les produits. En ce qui concerne les propriétés antisalissures, elles se conservent pendant 3 ans. Cette durée est valable dans les conditions d'exposition (vitesse des courants assez lente et non permanente).

Le pouvoir antisalissures des métallisations zinc est encore important après 20 mois d'exposition dans ces mêmes conditions.

\section{b) Comportement EN IMMERSION-Émersion.}

Nous retrouvons les mêmes phénomènes généraux qu'en immersion prolongée, mais à plus longue échéance.

Les meilleures sont une nouvelle fois :

Les bonnes peintures vinyliques dont certaines sont encore exposées après 5 ans le cloquage restant le principal phénomène de corrosion.

Les métallisations au zinc, malgré que l'usure du zinc soit encore plus manifeste qu'en immersion prolongée.

Les métallisations au zinc + peinture vinylique qui sont exceltentes.

Toutefois, 1 bitumineux et 1 caoutchouc ont atteint 4 ans d'exposition sans corrosion intense.

Les finitions antifouling supportent assez mal l'alternance d'exposition; à un craquellement généralisé succède un manque d'adhérence et des piqûres de rouille.

\section{c) Comportement a L'Air SALIN ET AUX EMbruns}

Cette exposition est beaucoup moins agressive que les précédentes :

- 1 bitumineux s'est comporté convenablement pendant 4 ans;
- 3 caoutchoucs ont atteint 4 ans; l'un est encore en essais après 5 ans $1 / 2$;

- 1 glycérophtalique est resté également 4 ans en essais;

- Les bonnes peintures vinyliques résistent au moins 6 ans sans corrosion active;

-- Les métallisations au zinc ou aluminium sont excellentes;

- Certains produits à la poudre de zinc sont également très bons.

G'est-à-dire que le choix reste assez grand pour les protections des superstructures.

\section{d) Essais spéctaux.}

Les résultats des essais spéciaux confirment pleinement la supériorité des peintures à base de résines vinyliques.

- Sur des éprouvettes tubulaires, les peintures se comportent mieux que sur les éprouvettes sous forme de plaques. Ces dernières soumettent les revêtements à d'importants effets de bords qui en hâtent l'altération; les arêtes vives doivent être supprimées.

- L'étude de l'influence de l'épaisseur des films vinyliques montre l'importance de ce facteur sur le cloquage. Le passage de l'épaisseur du film de 50 à $140 \mu$ améliore la tenue dans le rapport de 1 à 2 . De cette étude, il ressort qu'un système vinylique, pour être efficace, doit avoir une épaisseur de l'ordre de 180 microns.

- Les conditions d'essais en circuit hydraulique sur modèle réduit semblent plus dures que toutes les autres; il faut tenir compte des grandes vitesses de circulation d'eau, des couples existants entre métaux différents et surtout des effets de bord importants sur petites pièces.

- Les bonnes peintures vinyliques sont compatibles avec la protection cathodique sous des potentiels ne dépassant pas $-1200 \mathrm{mV}$ par rapport à l'électrode au calomel. Les peintures qui cloquent le plus facilement sous cet essai, cloquent le plus facilement dans la Rance. 


\section{B. - COMPORTEMENT DES MÉTAUX NOBLES}

\section{I. - PROGRAMME ET MOYENS D'ESSAIS}

Le programme d'essais des métaux nobles a porté sur leurs comportements aux conditions sévères d'utilisation.

Ce programme a été complété par l'étude des courbes de polarisation des métaux et des essais de polarisation cathodique effectués en eau-vive à Saint-Servan sous la conduite du S.E.P.O. du Gaz de France.

Des essais en flexion alternée et des essais de soudabilité ont également complèté ces études.

\section{a) Groupes D'essais de Matériaux : (G.E.M.)}

Les groupes G.E.M. (fig. 14) sont deux groupes, modèle réduit au $1 / 18^{\circ}$, représentant les groupes bulbes de l'usine de la Rance. Ces groupes sont montés en circuit fermé sur une citerne $(80000 \mathrm{I})$ contenant de l'eau de mer naturelle, renouvelée tous les trois jours afin de

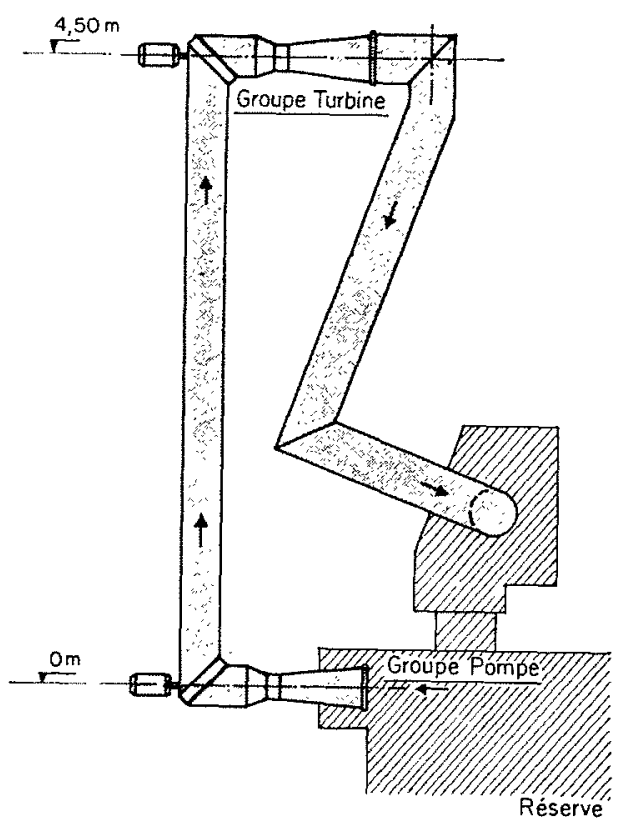

FIG. 14

G.E.M. lui conserver ses caractéristiques (en particulier : oxygénation).

L'un des groupes fonctionne en pompe, l'autre en turbine. Le cycle des essais comporte 8 jours de marche, suivis de 8 jours d'arrêt avec vidange du conduit.

Les essais des métaux nobles portent principalement sur les pales et les ceintures des groupes, dont les autres éléments sont constitués de la façon suivante :

- Distributeurs recouverts de peinture vinylique: Corps en acier ordinaire. Directrices en acier ordinaire pour l'un des groupes, en acier semi-inoxydable, $4 \%$ de chrome, pour l'autre.

- Moyeux de roue : En acier inoxydable $18 / 8 \mathrm{Mo}$, ou en acier ordinaire peint.

- Les arbres : En acier inoxydable à $13 \%$ de chrome puis en $18 / 8$.

- Le reste des machines est en acier ordinaire recouvert de peinture vinylique.

\section{b) Observations et Relevés.}

Tous les mois les groupes G.E.M. étaient démontés pour examen et photographies. Pour évaluer commodément le comportement de ces matériaux et leur évolution dans le temps, des répliques des états de surface ont été effectuées périodiquement à l'aide d'un vernis cellulosique. Des radiographies et des examens de coupes en fin d'essais complétaient nos observations.

\section{II. - RESULTATS DES ESSAIS}

Les essais ont été menés sur 15 nuances d'acier inoxydable dans la gamme allant de 13 à $24 \%$ de chrome et sur 4 bronzes d'aluminium.

La nature de ces divers matériaux apparaît sur le tableau II. 


\section{TAbleaU II}

\begin{tabular}{|c|c|c|c|c|c|c|c|c|c|}
\hline \multicolumn{2}{|c|}{ CATÉGORIES } & C & $\mathrm{Cr}$ & $\mathrm{Ni}$ & $\mathrm{Cu}$ & Mo & $\mathrm{AI}$ & $\mathrm{Fe}$ & $\mathrm{Mn}$ \\
\hline \multirow{5}{*}{ 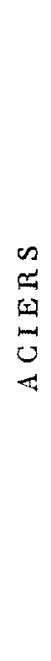 } & Semi-inoxydable. . . . . & 0,1 & 4 & 1 & & 0,2 & 1 & & \\
\hline & Martensitiques à $13 \% \mathrm{Cr}$ & $\begin{array}{l}0,1 \\
0,1 \\
0,1 \\
0,1\end{array}$ & $\begin{array}{l}13,1 \\
13,3 \\
12,8 \\
12,6\end{array}$ & $\begin{array}{l}1,3 \\
2,5 \\
0,2 \\
1,1\end{array}$ & & $\begin{array}{l}1 \\
1\end{array}$ & & & $\begin{array}{l}0,6 \\
0,9\end{array}$ \\
\hline & Martensitiques à $17 \% \mathrm{Cr}$ & $\begin{array}{l}0,2 \\
0,1 \\
0,1 \\
0,05\end{array}$ & $\begin{array}{l}16 \\
17 \\
17 \\
16,7\end{array}$ & $\begin{array}{l}3,4 \\
4 \\
4 \\
5,4\end{array}$ & $\begin{array}{l}1,4 \\
4 \\
2,5\end{array}$ & 1,5 & & & $\begin{array}{l}1,1 \\
0,4 \\
0,4 \\
0,7\end{array}$ \\
\hline & Austéno-ferritiques. . . . . & $\begin{array}{l}0,1 \\
0,1 \\
0,05\end{array}$ & $\begin{array}{l}24 \\
20 \\
20\end{array}$ & $\begin{array}{l}7,5 \\
7,5 \\
7,5\end{array}$ & $\begin{array}{l}0,6 \\
1,5\end{array}$ & $\begin{array}{l}2 \\
3 \\
2,4\end{array}$ & & & $\begin{array}{l}0,6 \\
0,5\end{array}$ \\
\hline & Austénitiques. . . . . . . . & $\begin{array}{l}0,04 \\
0,04\end{array}$ & $\begin{array}{l}18 \\
18 \\
18 \\
18\end{array}$ & $\begin{array}{r}8 \\
14 \\
8 \\
11\end{array}$ & & $\begin{array}{l}2,5 \\
3 \\
2,5\end{array}$ & & & \\
\hline \multirow{2}{*}{ 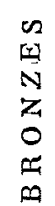 } & Cupro-Aluminium. . & & & $\begin{array}{l}5,1 \\
6,3 \\
0,3\end{array}$ & $\begin{array}{l}79,4 \\
79 \\
\text { Bal. }\end{array}$ & & $\begin{array}{r}9,5 \\
8,7 \\
10,4\end{array}$ & $\begin{array}{l}4,2 \\
4,5 \\
3,5\end{array}$ & $\begin{array}{l}1,8 \\
1,5\end{array}$ \\
\hline & $\begin{array}{c}\text { Cupro-Manganèse- } \\
\text { Aluminium. . . . . }\end{array}$ & & & 1,5 & Bal. & & 8,4 & 2,7 & 12,6 \\
\hline
\end{tabular}

a) Comportement SUR LES GROUPES G.E.M.

L'installation a été mise en service en octobre 1956; elle totalise à ce jour 17000 heures de marche.

Les aciers semi-inoxydables ètaient peints. Après deux ans d'essais, certaines parties qui avaient été mises à nu accidentellement étaient très attaquées.

Aciers martensitiques $\dot{a}^{"} 13 \%$ de chrome (fig. 15 et 16).

Ces aciers ont été essayés sous forme de pales, de ceintures de roue et d'arbres.

Les premiers points de corrosion apparaissent à $200 \mathrm{~h}$. La corrosion par pitting se traduit par des piqûres constituées d'un microtrou à peine apparent communiquant avec une caverne intérieure. Le nombre de ces piqûres augmente rapidement jusqu'à $3000 \mathrm{~h}$. Les défauts préexistants sont le siège d'attaques très marquées.

En plus de ces piqûres, nous avons noté des attaques très importantes sur les arbres aux portées sur palier's graphités (aération différentielle). Après $10000 \mathrm{~h}$, certains points d'attaques étaient profonds de $6 . \mathrm{mm}$.
Deux pales en acier à $13 \%$ de chrome et $1 \%$ de molybdène ont été en essais pendant $5700 \mathrm{~h}$. Leur comportement est meilleur que celui des pales à $13 \%$ de chrome sans molybdène. Les attaques apparaissent à plus longue échéance $(300 \mathrm{~h})$ et se développent moins rapidement. Les défauts de surface sont également le siège de corrosion particulière.

Aciers martensitiques $\dot{a} 17 \%$ de chrome $\dot{a}$ durcissement structural. (fig. 17 et 18 ).

Leur comportement est de beaucoup supérieur à celui des $13 \%$ de chrome.

Après $2600 \mathrm{~h}$, les pales de cette nuance sans molybdène ne présentent que quelque piqûres très peu profondes; par contre, la corrosion s'est installée dans les zones affectées par les défauts de coulée.

Une ceinture de cette même nuance était encore intacte à $3000 \mathrm{~h}$ de service. Après $6000 \mathrm{~h}$, elle présente des petits points de rouille foisonnante cachant des micropiqûres peu profondes.

Deux pales en acier à $17 \%$ de chrome et $1,5 \%$ de molybdène n'ont donné après $7700 \mathrm{~h}$ qu'une très légère prise à la corrosion. Les quel- 


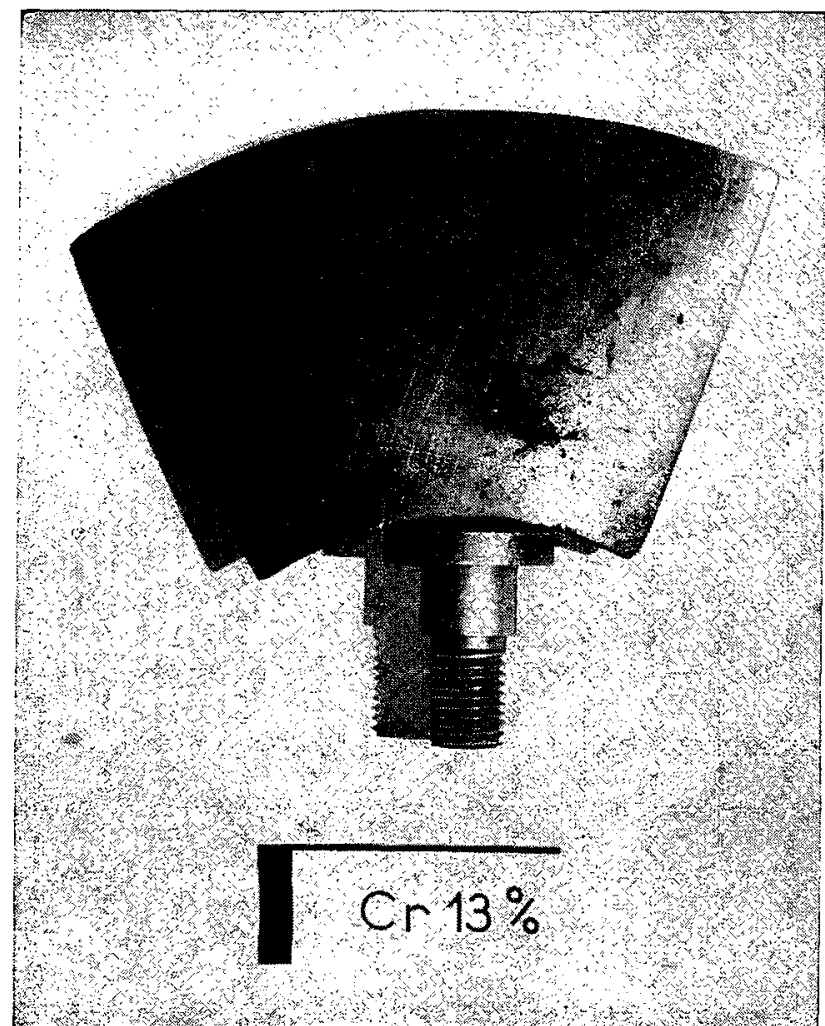

Fia. 15

Après 3500 heures de fonctionnement sur G.E.M. plus 1 mois d'immersion.

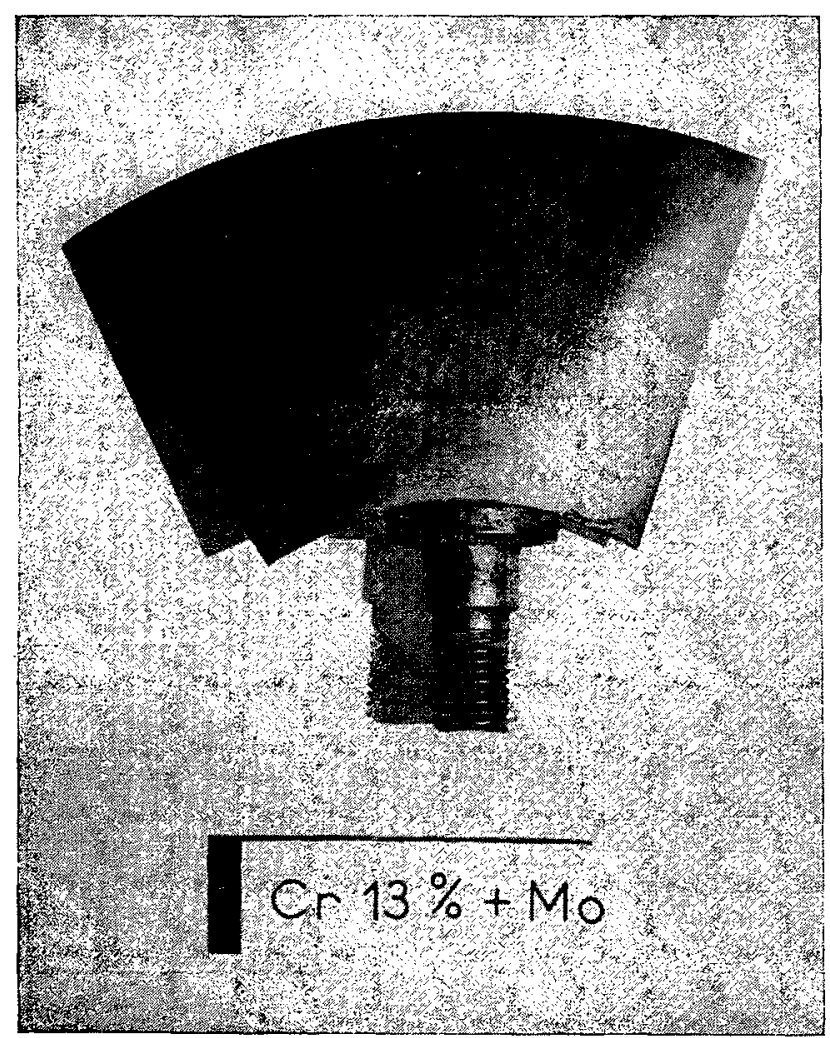

Fig. 16

Après 5700 heures de fonctionnement sur G.E.M.

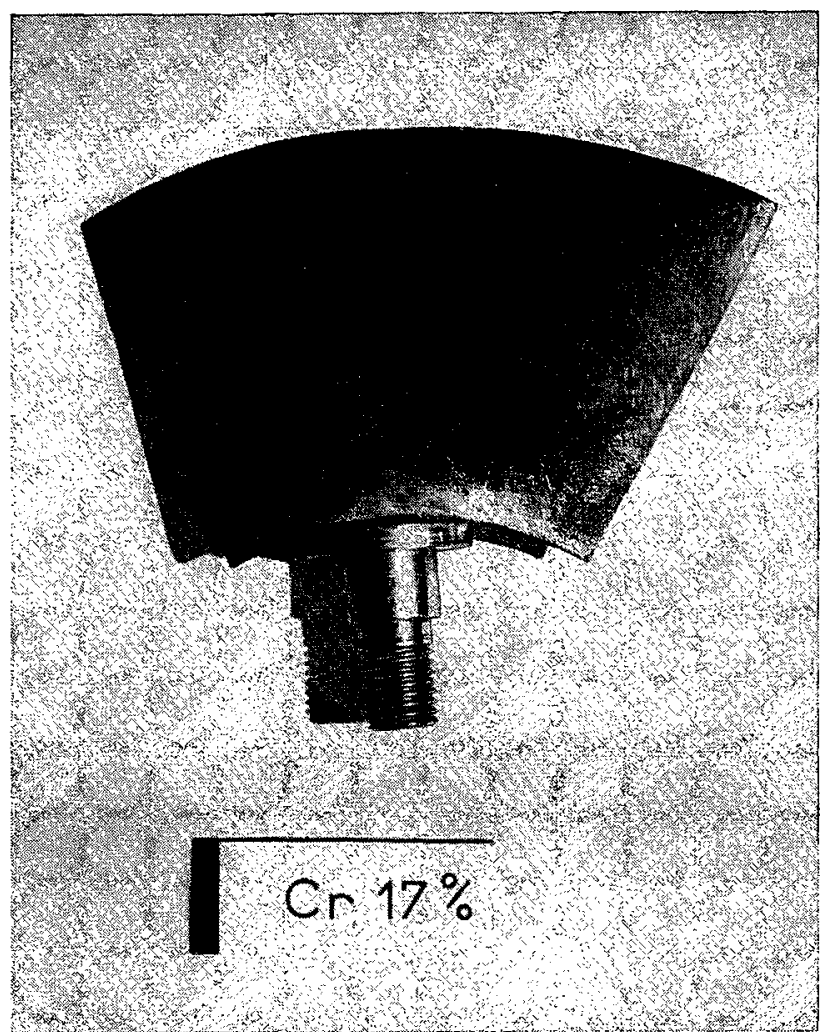

FIG. 17

Après 2600 heures de fonctionnement sur G.E.M. plus 5 mois d'immersion.

ques points d'attaque superficielle existants ont apparu à $4000 \mathrm{~h}$ et sont restés sans évolution visible. L'état de surface de ces pales était très soigné.

Aciers austéno-ferritiques ( $20 \%$ de $\mathrm{Cr}$ ).

D'une manière générale, les pales et ceintures en cette nuance n'ont pas donné prise à la corrosion après $2500 \mathrm{~h}$ d'essais pour les pales et $8500 \mathrm{~h}$ pour la ceinture.

La présence de défauts de coulée ne paraît pas avoir une influence aussi grande que sur les aciers à teneur en chrome moins élevée.

Aciers austénitiques (18\% de $\mathrm{Cr}$ ).

Une ceinture en acier ordinaire doublée d'acier austénitique s'est remarquablement comportée pendant $2500 \mathrm{~h}$ Un moyeu de roue est intact après $7700 \mathrm{~h}$.

Leś arbres chemisés en $18 / 8$ présentent après $7000 \mathrm{~h}$ de service quelques points d'attaque sur les portées des paliers graphités (aération).

\section{Cupro-Aluminium (Al $10 \%-\mathrm{Ni} 1 \%$ ).}

Cette nuance a été sensible à la cavitation, dont les traces ont été observées après $1800 \mathrm{~h}$ de fonctionnement et ont continué à s'accentuer. 


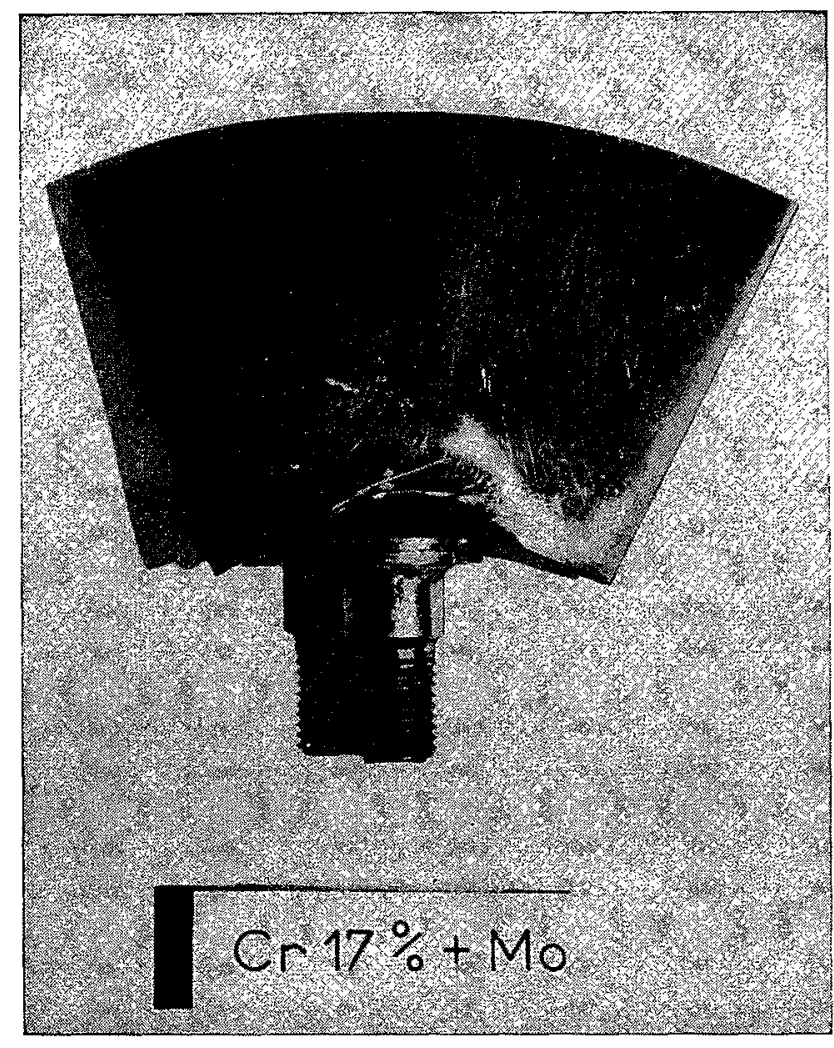

FIG. 18

Après 7700 heures de fonctionnement sur G.E.M.

\section{Cupro-Aluminium (Al $9 \%$ - Ni $5 \%$ ) (fig. 19).}

Très bonne tenue à la corrosion des pales de cet alliage après $6600 \mathrm{~h}$ de service sur les groupes GEM. Toutefois, des traces d'usure dues à l'abrasion dans les zones cavitantes sont apparues en fin d'essai.

\section{Cupro-Manganèsc-Aluminium (fig. 20).}

Les pales et la ceinture réalisées suivant cette nuance étaient recouvertes de nombreuses petites piqûres après $2000 \mathrm{~h}$ d'essai. Après $5000 \mathrm{~h}$ la ceinture présentait des zones d'usure par abrasion à l'endroit du passage des pales.

\section{b) Essais complémentaires.}

Après expiration du cycle d'essai G.E.M., une pale de chacun des matériaux était passivée puis immergée en Rance. Alors que les aciers austéno-ferritiques ont été retirés après 158 jours sans trace de corrosion apparente, l'acier à $17 \%$ de chrome présentait quelques points de rouille foisonnante, localisés sur les défauts de coulée. Quant aux aciers martensitiques, ils présentaient après 1 mois d'immersion de nombreuses et profondes piqûres.

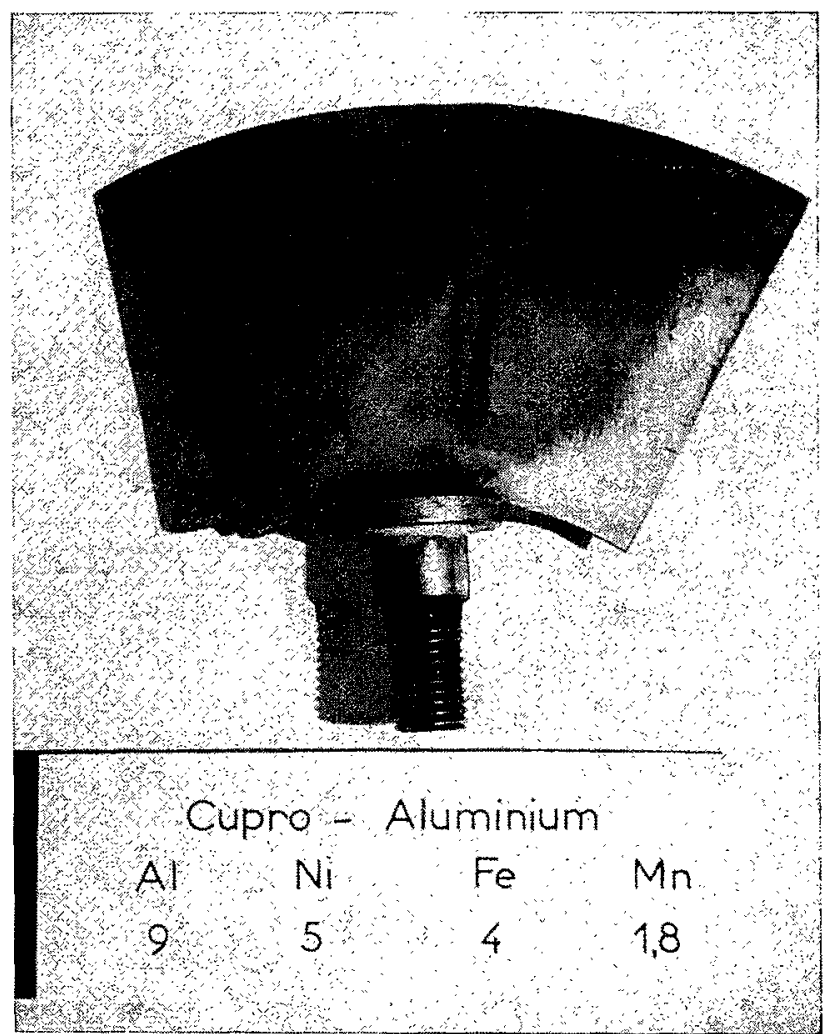

Fig. 19

Après 6600 heures de fonctionnement sur G.E.M.

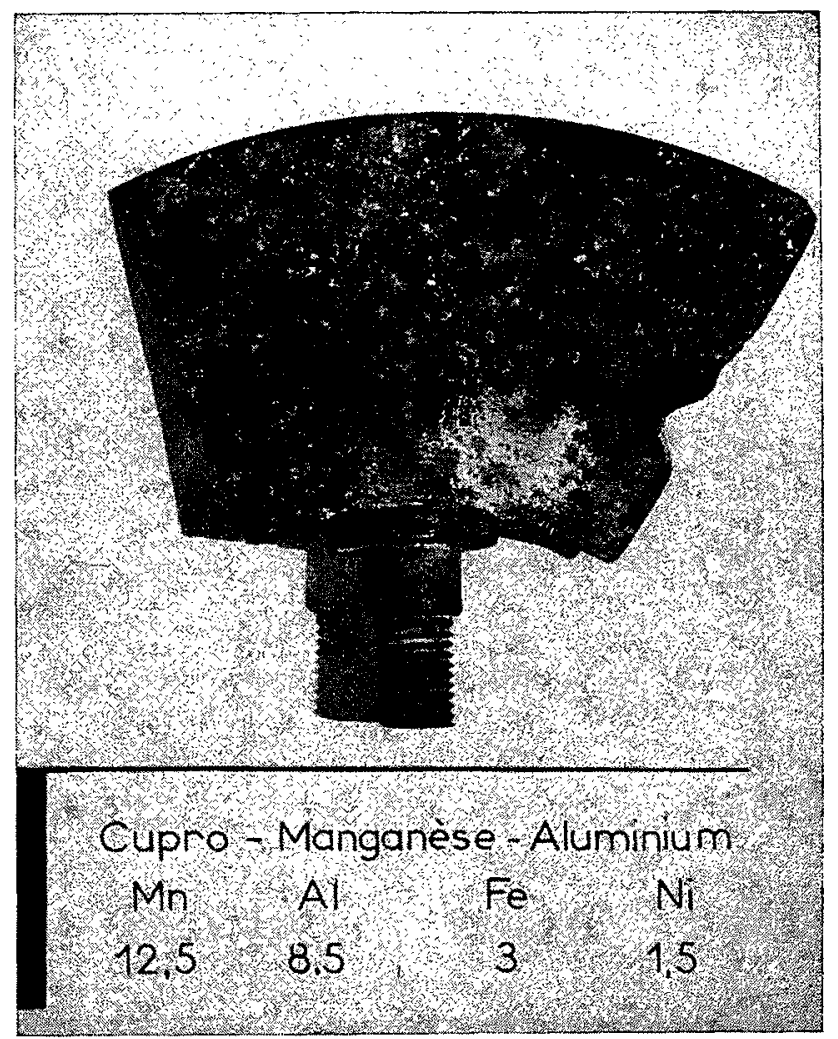

Fig. 20

Après 2400 heures de fonctionnement sur G.E.M. plus 5 mois d'immersion. 
Les Cupro-Manganèse-Aluminium se sont couverts de pustules d'oxyde « rosette de rouille » dès les premières semaines d'immersion.

Les Cupro-Aluminium d'une teneur en fer et nickel inférieure à $3 \%$ se sont également couverts de pustules blanchâtres sous lesquelles le métal est rouge cuivre (désaluminisation).

L'importante corrosion observée sur certains matériaux immergés ne s'était pas manifestée sur G.E.M., probablement grâce à la proximité d'acier moins noble réalisant une protection cathodique efficace.

Les essais de soudabilité ont montré la nécessité de réaliser des soudures sans inclusions d'acier ordinaire et totalement étanches dans les cas de doublage pour éviter les pénétrations d'eau de mer entre acier ordinaire et revêtement.

Des essais de résistance en flexion alternée sous 100 millions de cycles montrent qu'en eau de mer la limite de fatigue des aciers inoxydables subit un abaissement beaucoup plus important que celle des cupro-aluminium.

La limite de fatigue d'un martensitique à $17 \%$ de chrome passe de $35 \mathrm{~kg} / \mathrm{mm}^{2}$ dans l'air à 17 dans l'eau de mer; alors qu'un cupro-aluminium passe de $18 \mathrm{~kg} / \mathrm{mm}^{2}$ dans l'air à 15 en eau de mer (eśsais conduits identiquement).

Ces essais d'immersion ont également mis en relief la sensibilité des aciers inoxydables à la corrosion par «aération différentielle .

\section{CONCLUSION}

Les- essais effectués depuis 1955 par le Laboratoire d'essais du S.E.U.M. sous. l'égide du "Groupe corrosion》 ont apporté des connaissances extrêmement précieuses et indispensables pour la bonne tenue du matériel à la corrosion marine.

Elles peuvent être résumées très brièvement de la façon suivante :

\section{REVÊteMENTS ANTICORROSIFS.}

Les meilleurs résultats obtenus l'ont été avec certaines peintures vinyliques, dont l'une donne particulièrement satisfaction, et ne semble pas nécessiter de réfection avant 5 ans.

A l'air salin, on peut espérer une tenue plus longue.

L'efficacité des finitions anti-fouling est de l'ordre de 3 ans.

Les métallisations zinc au voisinage d'acier inoxydable ou bronze sont à proserire, malgré leur bon comportement intrinsèque.

Cependant, des précautions indispensables doivent être prises pour obtenir ces résultats, en particulier : bon état de surface, excellente préparation des fonds, suppression des angles vifs et contrôle de l'épaisseur du film qui, pour des systèmes vinyliques, doit être de l'ordre de 180 microns.

\section{Métaux inoxydables.}

Compte tenu des diverses caractéristiques: résistance à la corrosion, limite de fatigue en eau de mer, facilité de mise en œuvre, soudabi- lité... on peut extraire de ces essais trois alliages intéressants :

- L'acier inoxydable $17 \%$ de chrome à durcissement structural, dont la bonne tenue à la corrosion est encore améliorée par l'addition de $2 \%$ de molybdène. Cet alliage a de très bonnes caractéristiques mécaniques, une bonne soudabilité moyennant quelques précautions. Toutefois, les défauts de coulée provoquent des amorces de corrosion; de plus il est sensible à la corrosion par aération différentielle.

- L'acier austénitique $18 \%$ de chrome - $11 \%$ de nickel et $3 \%$ de molybdène, pour sa très bonne tenue à la corrosion marine. Toutefois, il est également sensible à la corrosion par aération différentielle.

- Les Cupro-Aluminium à faible teneur en manganèse, sous réserve d'une teneur en fer et en nickel de 3 à $5 \%$ afin d'éviter le phénomène de désaluminisation qui peut apparaître en eau vive très oxygénée. De bonne soudabilité, ils présentent quelques difficultés de coulée pour de très grosses pièces.

Enfin, une dernière étape précédant la construction définitive des groupes de la Rance a été la mise en service, en octobre 1959, d'un groupe expérimental dans une écluse désaffectée du port de Saint-Malo. Usine-pilote dont le comportement et la tenue à la corrosion marine confirment pleinement les résultats des essais et l'intérêt des solutions envisagées. 


\section{I S CUS S I O N}

Président : M. Ratr

M. le Président remercie M. Licheron d'avoir excellemment résumé six et presque sept ans de travaux d'un groupe de techniciens très important, et ouve la discussion.

M. Raud demande quelle peut être l'action du sable, quand la mer en contient, sur les métaux.

M. Licheron répond qu'il a parlé, dans sa communication, des effets abrasifs du sable, en particulier sur les cupro-aluminiums, à propos des essais sur groupes G.E.M.

D'autre part, la teneur en sable de l'eau de la Rance est inférieure à celle de l'eau de la citerne du groupe G.E.M. et l'effet du sable sur les groupes industriels sera réduit, ce qui est vérifié même sur les peintures exposées en Rance.

M. Beltremieux demande si le chiffre de 180 microns indiqué pour les peintures vinyliques s’appliquerait à des structures de génie civil soumises uniquement à l'air marin et aux embruns, ou si l'on pourrait descendre plus bas pour une protection de très longue durée (ponts, ouvrages de service ou un viaduc entre la terre et une ille).

M. Lrchenon rappelle qu'il s'agit d'un système vinylique multi-couches composé :

- d'une couche d'impression phosphatante;

- d'un certain nombre de couches ( 3 en moyenne) anti-corrosion, dont le liant est à base de résine vinylique, auquel sont intimement incorporés des pigments inhibiteurs qui peuvent être du chromate de zinc, du minium de plomb, du plombate de calcium;

- de deux couches de finition, donnant la couleur ou, dans les cas d'immersion, ayant un pouvoir antisalissures. La première couche a une épaisseur de 15 microns; chacune des couches inhibitrices a une épaisseur de l'ordre de 30 à 40 microns. Dans I'atmosphère marine; une épaisseur totale de 150 microns, sera optimale; elle sera obtenue par la couche d'impression, deux couches anticorrosion, deux couches de finition.

Sur des remarques de M. Beltremieux, M. Licheron précise, d'une part, que l'on n'a pas intérêt à avoir une protection trop épaisse et, d'autre part, qu'il a essayé certaines peintures à haute viscosité, mais que les essais, datant de moins d'un an, ne peuvent conduire à des résultats définitifs : ces peintures sont encore «très belles », mais il a essayé d'autres peintures qui le sont restées durant 4 ans.

M. Beltremeux demande si l'application de peinture vinylique sur des structures de génie civil n'est pas trop sensible à l'humidité, à l'application, à l'entretien.

M. Lrcheron répond que les peintures vinyliques ne peuvent être appliquées sur surface mouillée et qu'aucun produit ne donnera de très bons résultats dans ce cas. Cependant, les applications conduites sur chantier donnent satisfaction; tel en témoignera M. Sanhes qui parlera des résultats obtenus sur le groupe expérimental de Saint-Maló.

D'autre part, l'un des avantages des peintures vinyliques est leur temps de séchage très rapide: 2 heures pour la première couche, 20 à 30 minutes pour les couches suivantes.

M. Lreber pose les questions suivantes :

10) Quelle est l'importance des arrondis à prévoir sur les pièces? $\left.2^{\circ}\right)$ Comment tiennent les réparations de peinture?

M. Licheron répond, pour la première question, qu'il n'a pas fait d'essais systématiques, mais que, d'après les essais effectués sur le groupe de Saint-Malo, on a admis que les arrondis devalent avoir environ $5 \mathrm{~mm}$ de rayon (minimum de sécurité).

Pour répondre à la deuxième question, M. LichenoN dit que les réparations avec les peintures vinyliques sont facilement réalisables. Des retouches faites sur une protection ayant été exposée préalablement 4 ans se sont très bien comportées.

Certaines retouches ne nécessitent pas la reprise complète du système si elles sont entreprises assez tôt. Elles peuvent répartir des couches inhibitrices.

D'autre part, afin de limiter ces retouches, le problème du transport et du montage des pièces doit être examiné avec soin. M. Sanhes en parlera dans sa communication.

M. VASSAL pense, au sujet des limites de fatigue, que pour les martensitiques à $17 \%$ de chrome, la valeur donnée par M. Licheron est un peu faible. Des essais effectués au laboratoire de la S.F.A.C. en eau de mer, sur ces aciers normalisés, ont donné $21 \mathrm{~kg}$.

M. Licheron a déjà souvent constaté que lorsqu'un chiffre de limite de fatigue était annoncé, sa valeur était souvent contestée par les métallurgistes, et ce, toujours dans le même sens.

M. Vassal ajoute que, suivant le mode de prélèvement des éprouvettes, le chiffre peut différer. Les Suédois à Avesta ont trouvé à peu près les mêmes résultats pour cet acier.

M. Licheron ne peut donner de précision sur ce point, car ce n'est pas lui qui a fait les essais.

M. Berthier apporte quelques précisions au sujet des moyens d'évaluation de la valeur des peintures et revêtements à base de corps organiques, dits plastiques. Il faut dans des études de ce genre, èvaluer séparément les constantes intrinsèques de la matière de base et les caractéristiques technologiques des peintures et revêtements réellement mis en œuvre, qui peuvent être assez différents de la matière de base.

Par exemple, une donnée importante est le coefficient d'absorption d'eau après une immersion très prolongée et après des variations importantes de pression et d'humidité. Pour les matières pures, les producteurs ne donnent pas toujours volontiers ces coefficients, mais on peut assez facilement les mesurer soi-même. On constate alors que ces coefficients diffèrent considérablement d'une matière à l'autre. Des corps qui, à première vue, paraissent peu hydrophiles et, du point de vue électrique, peu conducteurs, ont un coefficient d'absorption d'eau non négligeable, par exemple, les caoutchoues à l'état pur. Les caoutchoues industriels, qui contiennent souvent des proportions énormes de charges minérales peuvent absorber encore plus d'eau.

M. Licheron rappelle que l'ean de mer n'est pas une simple solution d'électrolytes. C'est un milieu vivant qui subit des modifications provoquées par le métabolisme des organismes. Ces modifications locales sont dues aux végétaux dont les principales sont la production d' $\mathrm{O}^{2}$ et l'élévation du $\mathrm{pH}$, aux animaux dont les actions respiratoires consomment de $\mathrm{l}^{\prime} \mathrm{O}^{2}$ et produisent $\mathrm{du} \mathrm{CO}^{\circ}$, aux bactéries (formation de $\mathrm{H}^{2} \mathrm{~S}$ ).

Ainsi, les résultats obtenus en laboratoire, par exemple 
au brouillard salin, en cycle accéléré, peuvent être différents des résultats des cycles en immersion dans les conditions naturelles d'essais.

M. Benthier ajoute, à propos de gaz, que la détermination de la teneur en eau d'un plastique doit être accompagnée de la mesure de perméabilité aux gaz, en particulier $\mathrm{CO}^{2}, \mathrm{O}^{2}$ et vapeur d'eau. Là, on trouve des résultats très curieux, et les coefficients de perméabilité sont très différents suivant la nature de ces plastiques.

Par exemple, les polyéthylènes, qui sont des paraffines à longues chaînes, sembleraient à première vue hydrophobes. Pourtant, ils sont perméables à la vapeur d'eau. Ils sont très perméables au $\mathrm{CO}^{2}$, à tel point que si l'on remplit une bouteille en polyethylène avec du $\mathrm{CO}^{2}$ pur et qu'on la bouche bien, cette bouteille s'aplatit progressivement et elle est à peu près complètement aplatie au bout d'une semaine. La pression du $\mathrm{CO}^{2}$ qui reste, égale alors la pression partielle de ce gaz dans l'atmosphère extérieure.

Par contre, le même corps est très peu perméable à $\mathrm{O}^{\varepsilon}$ et $\mathrm{Ar}^{2}$, et par conséquent à l'air sec.

De même, on sait qu'on ne peut pas gonfler des pneus au $\mathrm{CO}^{2}$, ce qui serait pourtant tellement pratique.

$\mathrm{Au}$ point de vue de la protection du fer contre la corrosion, ces particularités sont fâcheuses, car l'eau et les ions qu'elle entraîne, et le $\mathrm{CO}^{2}$ jouent un rôle important dans la corrosion. Le Centre de Recherches que dirige M. Berthier a eu à résoudre des problèmes de protection d'aciers de précontrainte de barrages. Il a fallu faire des gaines multiples pour obtenir a la fois une bonne imperméabilité et une bonne tenue à l'érosion mécanique.

M. Licheron dit que ces revêtements ont nue mauvaise adhérence sur le métal de base et, en cas d'accident, la corrosion gagne et décolle le revêtement. M. Licheron l'a noté dans la majeure partie des cas.

M. Berthier dit qu'effectivement ces revêtements sont souvent peu adhérents au métal. Il faut alors traiter la surface métallique et $y$ disposer un mince film de substance organique, telle que l'araldite, qui adhère à la fois au métal et aux revêtements plastiques subséquents.

En tout cas, toute mesure de perméabilité aux différents agents, eau, gaz, etc., doit être complétée par une mesure de conductibilité électrique, sous réserve que la tension électrique utilisée pour la mesure soit très faible, car il ne s'agit pas du tout de mesurer la tension de claquage sous haute tension, mais de mettre en évidence les phénomènes électrolytiques.

M. le Président donne, pour clore cette discussion, la parole à M. Raurer. Il est bien certain que chacun des points soulevés pourrait être discuté pendant longtemps et la discussion sur tous ces problèmes sur le plan industriel reprendra à la suite du mémoire de M. Sanmes.

M. Rabier demande si des essais ont été faits sur des revêtements à base de polyamide (rilsan, nylon).

M. Licheron dit qu'il a été fait un essai d'application de rilsan sur une hélice de bateau en fonte, qui a fonctionné pendant un an. Après ce laps de temps, les extrémités des pales étaient mises à nu, mais il y avait une très bonne tenue du revêtement au niveau du moyeu. Cependant, ce revêtement nécessite un pré-chauffage qui, pour le cas de grosses pièces, est un lourd handicap.

M. le Président remercie M. Licheron pour sa communication et les personnes qui ont pris part à la discussion.

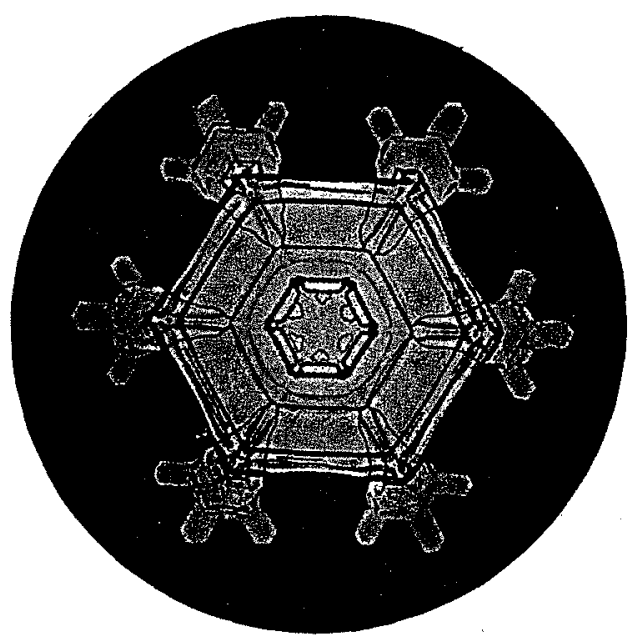

